\title{
Tobacco Transgenic for the Flax Rust Resistance Gene $L$ Expresses Allele-Specific Activation of Defense Responses
}

\author{
Donna Frost,,${ }^{1,3}$ Heather Way, ${ }^{2}$ Paul Howles, ${ }^{1}$ Joanne Luck, ${ }^{1}$ John Manners, ${ }^{2}$ Adrienne Hardham, ${ }^{3}$ Jean \\ Finnegan, ${ }^{1}$ and Jeff Ellis ${ }^{1}$ \\ ${ }^{1}$ CSIRO Plant Industry, Cnr Clunies Ross Street and Barry Drive, Acton ACT 2601, Australia; ${ }^{2}$ CSIRO Plant Industry, \\ Queensland Bioscience Precinct, 306 Carmody Road, St Lucia QLD 4067, Australia; ${ }^{3}$ Research School of Biological \\ Sciences, Australian National University, Canberra ACT 0200, Australia
}

Submitted 27 August 2003. Accepted 11 October 2003.

\begin{abstract}
Tobacco was transformed with three different alleles $(L 2$, $L 6$, and $L 10$ ) of the flax rust resistance gene $L$, a member of the toll interleukin-1 receptor, nucleotide-binding site, leucine-rich repeat (TIR-NBS-LRR) class of plant disease resistance genes. $L 6$ transgenics had a stunted phenotype, expressed several defense response genes constitutively, and had increased resistance to the fungus Cercospora nicotianae and the oomycete Phytophthora parasitica pv. nicotianae. $L 2$ and $L 10$ transgenics, with one exception for L10, did not express these phenotypes, indicating that the activation of tobacco defense responses is $L 6$ allelespecific. The phenotype of the exceptional $L 10$ transgenic plant was associated with the presence of a truncated $L 10$ gene resulting from an aberrant T-DNA integration. The truncated gene consisted of the promoter, the complete TIR region, and 39 codons of the NBS domain fused inframe to a tobacco retrotransposon-like sequence. A similar truncated $L 10$ gene, constructed in vitro, was transiently expressed in tobacco leaves and gave rise to a strong localized necrotic reaction. Together, these results suggest that defense signaling properties of resistance genes can be expressed in an allele-specific and pathogenindependent manner when transferred between plant genera.
\end{abstract}

Additional keywords: NBS-LRR resistance genes, plant defense.

Genetically determined resistance to pests and pathogens is used extensively in agriculture. Plant breeders have traditionally been restricted to sources of resistance genes from within the crop species or from closely related species. With the development of techniques to transform crop plants and the cloning of plant disease resistance genes, the potential sources of disease resistance have been considerably enlarged. In general, disease resistance genes act in a specific manner to provide resistance in a plant species against pathogen strains that carry corresponding avirulence genes. Resistance gene products are believed to have two principal roles. First, either directly or indirectly, they facilitate specific recognition of pathogen avirulence determinants, and second, following recognition, they activate cellular mechanisms in the host that lead to disease resistance. There are now several examples of effective genetic-

Corresponding author: J. Ellis; E-mail: jeff.ellis@ csiro.au engineering-based transfer of resistance genes between plant genera in the family Solanaceae (Tai et al. 1999; Whitham et al. 1996). The transgenes perform both recognition and defense activating functions against the same specific avirulent pathogen in different host genera. There are also examples, within the same plant family, when overexpression of resistance transgenes from one species in a second species has resulted in the constitutive activation of plant defense genes in the absence of avirulent pathogens, causing broad resistance to bacterial and fungal pathogens (Olroyd and Staskawicz 1999; Tang et al. 1999). These examples suggested that the crossspecies transfer of resistance transgenes, at least within the same plant family (Tai et al. 1999), may provide new gene-forgene resistance in some cases, while in others it has potential for the development of broad-range nonspecific resistance via deregulated activation of the plant defense response. More recently, transgenic studies have also shown that two different types of $R$ genes can reduce disease when transferred from one plant family to another (Hennin et al. 2001; Xiao et al. 2003).

As part of a study to examine the behavior of resistance genes transferred between plant families, we made transgenic tobacco plants (family Solanceae) containing the L2, L6, and $L 10$ alleles of the flax rust resistance gene $L$ (Ellis et al. 1999; Lawrence et al. 1995) from flax (family Linaceae). The flax $L$ gene is a member of the largest group of plant resistance genes, the nucleotide binding site-leucine rich repeat (NBSLRR) class, and confers gene-for-gene strain-specific resistance against the flax rust fungus, Melampsora lini. The $L$ gene belongs to the subgroup of NBS-LRR genes that encode an Nterminal domain called the toll interleukin-1 receptor (TIR) domain that has amino acid sequence similarity to the toll and interleukin-1 receptor proteins of Drosophila and mammals, respectively. Here, we show that expression of $L 6$ in tobacco gives rise to spontaneous activation of defense responses, increased resistance to two tobacco pathogens, and a stunted plant phenotype. However, activation of defense responses by the flax $L$ gene was found to be allele specific. With one exception (L10), out of 40 transgenic plants for each allele, only the $L 6$ allele and not the $L 2$ or $L 10$ alleles had this effect. The single $L 10$ plant, whose phenotype was more severe than any L6 transgenic, carried a full-length and a truncated $L 10$ gene. Transient assays indicate a role for the truncated form of $L 10$, which encodes essentially only the N-terminal TIR homology region of the L10 protein, in inducing the defense response in this single transgenic plant. 


\section{RESULTS}

Transgenic tobacco plants carrying

the $L 6$ rust resistance gene have a stunted phenotype.

An Agrobacterium strain carrying a binary vector containing a genomic clone of $L 6$ and a spectinomycin gene for plant selection was used to transform tobacco (Nicotiana tabacum). In several independent experiments, more than $50 \%$ of the spectinomycin-resistant plants recovered from the transformations developed a stunted phenotype in tissue culture (Fig. 1A and B). This phenotype was not seen in transformation experiments involving an "empty" vector (spectinomycin resistance gene only) or other non- $L$ gene constructs we have used for many previous tobacco transformation experiments. An identical phenotype was observed in tobacco transformed with a cDNA copy of $L 6$ that contained only the coding region of the gene expressed from the $35 \mathrm{~S}$ CaMV promoter. These visual characteristics of the $L 6$ tobacco transgenics became accentuated during subsequent clonal subculture steps, and plants frequently became chlorotic. Four transgenic plants, JL96-1, JL96-2, JL96-3, and J6-1 were selected for closer study and transferred to soil. $\mathrm{T}_{1}$ seeds were sown on solid tissue culture medium either with or without spectinomycin, the selectable marker for the transforming T-DNA. In the absence of spectinomycin and at about six weeks postgermination, it became evident that the stunted seedlings with downwardbending petioles, and the wild-type phenotype segregated among the progeny seedlings (Fig. 1B). In the presence of spectinomycin, the $T_{1}$ progeny segregated for green resistant seedlings and sensitive white seedlings. All resistant seedlings had the stunted phenotype, which indicated that the character was linked to the T-DNA. Analysis of 18 stunted and 4 wildtype $T_{1}$ segregants by polymerase chain reaction (PCR) demonstrated that the stunted phenotype and the L6 gene cosegregated. Reverse-transcription (RT)-PCR analysis of four stunted $\mathrm{T}_{1}$ progeny showed that the $L 6$ gene was expressed (data not shown). When $\mathrm{T}_{1}$ seeds were sown directly to soil, progeny also segregated for normal and stunted plants. Apart from a smaller size at the same age and delayed time to flower, the stunted plants were fertile and, in contrast to the chlorosis seen in tissue culture, soil-grown plants appeared otherwise normal. Transformed plants reached the same average height and flowered six to eight weeks later than control plants.

Transformation experiments with three L6 mutants showed that the stunted phenotype depended on the wild-type $L 6$ gene product. One mutant in the genomic clone of the $L 6$ gene was made by filling in a BamHI site in exon1, which causes a frame shift, a premature stop codon in exon1, and a truncated predicted gene product of only 162 residues compared with the 1,294 amino acid wild-type protein. A second mutant was made by filling in a HindIII site in exon 4 of a $35 \mathrm{~S}-L 6 \mathrm{cDNA}$ construct. This mutant introduces a frame shift and premature stop codon and is predicted to cause deletion of the $28 \mathrm{C}$-terminal amino acids from the predicted L6 protein. The third mutant was made by introducing a single base change into the 35S-L6cDNA construct that causes an amino acid substitution in the P-loop motif of the nucleotide binding domain (GMG GIGKT to GMGGIGTT) that is essential for resistance gene function (Dinesh-Kumar et al. 2000; Tao et al. 2000; Tornero et al.2002). Transgenic plants (20 or more) were recovered for each mutant $L 6$ construct, and all plants had a wild-type phenotype.

\section{The stunted phenotype is induced}

in tobacco transformed with $L 6$ but not with $L 2$ or $L 10$.

Tobacco was also transformed with cloned genomic fragments of two other $L$ gene alleles that share over $90 \%$ nucleic acid identity with $L 6$. The alleles, previously confirmed as functional by transformation of flax (Ellis et al. 1999), encode different rust resistance specificities in flax. A total of $40 \mathrm{~L} 2$ and 40 L1O transgenic tobacco plants were recovered in two different transformation experiments. In the first experiment involving only L2 and L10, all 20 L2 transgenics and 19 of 20 L10 transgenics were wild-type in appearance. In the second experiment, transformations with $L 2, L 6$, and $L 10$ were carried out simultaneously, and 20 transgenics were recovered for each allele. All $L 2$ and $L 10$ plants were wild-type, and 17 out of 20 L6 plants developed the stunted phenotype during tissue culture growth. DNA gel blot analysis of 12 L2 and 12 L10 plants chosen at random confirmed the presence of the $L$ transgene (data not shown). Furthermore, RNA gel blot analysis indicated that the absence of phenotype in the L2 (data not shown) and $L 10$ transgenics (Fig. 2B) was not correlated with lower $L$ gene transcript levels compared with $L 6$ plants.

The one exceptional L10 plant, called L10-A, was more stunted in its growth than any transgenic L6 plant (Fig. 1C). This difference was also seen in soil-grown plants. Cuttings taken from L10-A maintained in tissue culture were transferred to soil in a glasshouse. The soil-grown plants had crinkly, chlorotic leaves, poor vigor, and produced few flowers and only one or two mature seed capsules per plant. On tissue culture medium with no spectinomycin and at three weeks postgermination, $T_{1}$ progeny seedlings segregated for stunted phenotypes consistent with a 3:1 segregation ratio. After one
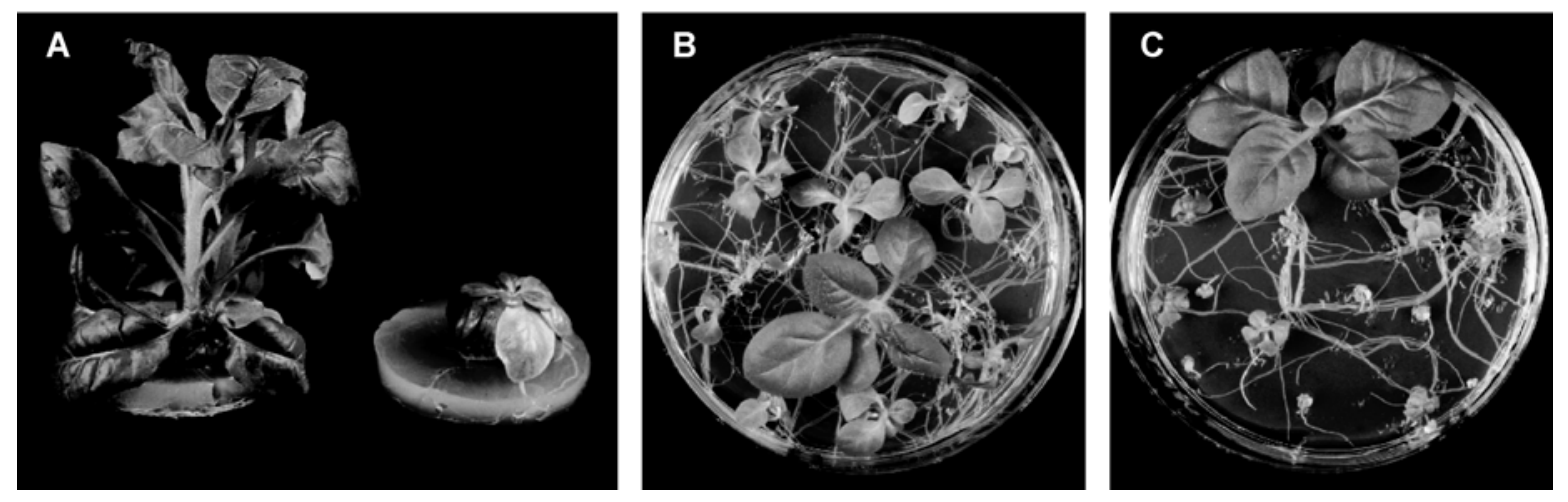

Fig. 1. Tobacco phenotypes associated with L6 and L10 transgenes. A, W38 (left) and JL96-1 (right) containing L6 and grown from shoot-tip cuttings on agar medium in sterile culture. Cuttings were transferred at the same time about 8 weeks prior to photography. B, $\mathrm{T}_{1}$ seedlings of JL96-1 growing on agar medium without spectinomycin segregate for stunted and normal plants. $\mathbf{C}_{1} \mathrm{~T}_{1}$ seedlings from the L10-A $\mathrm{T}_{0}$ transgenic plant growing on agar medium segregate for normal, stunted, and extremely stunted chlorotic seedlings. L10-A was the only plant out of the 40 L10 transgenics that had a stunted phenotype resembling the $L 6$ plants. 
or more weeks of further growth, it became clear that the stunted class was made up of two sorts of plants, stunted and extremely stunted plants (Fig. 1C), consistent with 2:1 ratio. Taken together, these data are consistent with the segregation of a single semidominant transgene locus in which the stunted plants are heterozygous and extremely stunted progeny are homozygous for the transgene. When spectinomycin was included in the medium, $\mathrm{T}_{1}$ seedling segregated for green and white seedlings with a ratio of $3: 1$, no wild-type plants were spectinomycin recovered, and all spectinomycin-resistant seedlings had a stunted or extremely stunted phenotype, which indicated that this phenotype was linked to the T-DNA and that the stunted and extremely stunted plants were probably hemizygous and homozygous for the transgene locus. A similar transgene dosage effect was not evident with L6 transgenics. The extremely stunted phenotype was lethal, and such seedlings did not develop further in tissue culture and died when transferred to soil.

\section{The phenotype of the L10-A plant is associated with a truncated $L 10$ gene containing mainly the TIR region.}

In an attempt to understand why the L10-A plant was abnormal, we performed DNA and RNA blot analysis of L10-A and normal $L 10$ transgenics. DNA blot analysis using a probe from the promoter region of the $L$ gene showed that all $12 L 10$ transgenics examined carried the $L 10$ gene on an approximately 7-kb EcoRI restriction fragment (Fig. 2A and data not shown), equivalent to the EcoRI fragment cloned into the binary plasmid used for transformation (Ellis et al. 1999). In addition, L10-A carried a smaller, approximately 5-kb fragment, suggesting that a truncated $L 10$ gene was present, possibly the result of an incomplete T-DNA integration (Fig. 2A). Further restriction mapping of genomic DNA of L10-A (data not shown) indicated that the truncated gene probably lacks the $3^{\prime}$ half (or more) of the coding region of L1O. Since only a single T-DNA locus segregated in the abnormal line, it is inferred that the full-length and the truncated $L 10$ gene are at the same T-DNA insertion site or at closely linked sites. RNA gel blot analysis indicated that the normal L1O trans- genics expressed a full-length $L 10$ transcript and that the stunted plant weakly expressed this transcript and a second substantially smaller transcript (Fig. 2B), which is consistent with the interpretation made from DNA blot analysis that this plant carried a normal and a truncated copy of $L 10$.

We used an RNAi strategy to demonstrate that the stunted phenotype of the L10-A line was due to the L10 transgene (either the full-length and truncated genes together or just the truncated gene) and not due to a T-DNA insertion mutation or tissue culture-induced mutation. Spectinomycin-resistant, stunted $\mathrm{T}_{1}$ progeny of L10-A (presumptive hemizygotes) were retransformed with an inverted repeat consisting of part of exon 1 of the $L 10$ gene expressed from the CaMV 35S promoter, and transformants were selected using a kanamycinresistance marker. This transformation was more difficult to achieve than transformation of normal tobacco because of the stunted and chlorotic character of the explant source used for transformation. Nevertheless, 12 independent transformants were recovered, and 10 of these had a wild-type phenotype in tissue culture and as soil-grown plants in the glasshouse. As a control for the possible selection of normal, tissue cultureinduced revertant cells during transformation and regeneration, we attempted to transform the stunted line with an empty vector that carried only the kanamycin-selectable marker, but no plants were recovered, probably due to the strong L10-A phenotype. However, in the absence of transformation and selection, we were able to regenerate 20 plants via tissue culture from leaf tissue of four different stunted $T_{1}$ progeny of L10-A. All regenerated plants retained the stunted phenotype, which indicated that the revertant plants recovered from the RNAi experiment were not due to selection for normal plants induced by tissue culture.

A portion of the truncated L1O copy was cloned and sequenced. The analysis demonstrated that the $L 10$ gene was truncated in exon 21,052 nucleotides downstream of the ATG translation initiation codon and was fused in-frame to a tobacco gene sequence with similarity to a retrotransposon gene. Translation of the cloned sequence predicts an amino acid sequence that contains the TIR region and 39 amino acids of the NBS region of $L 10$ (the truncation occurring approxi-
A

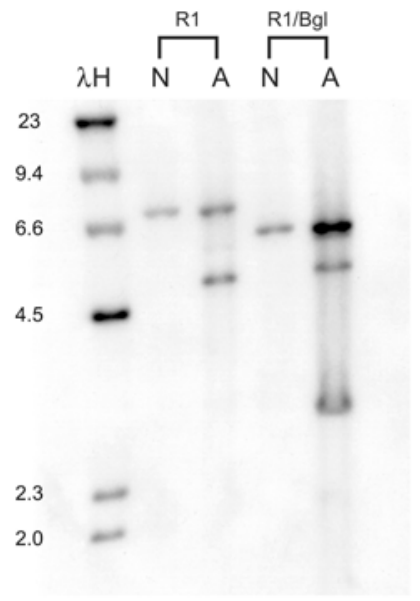

B

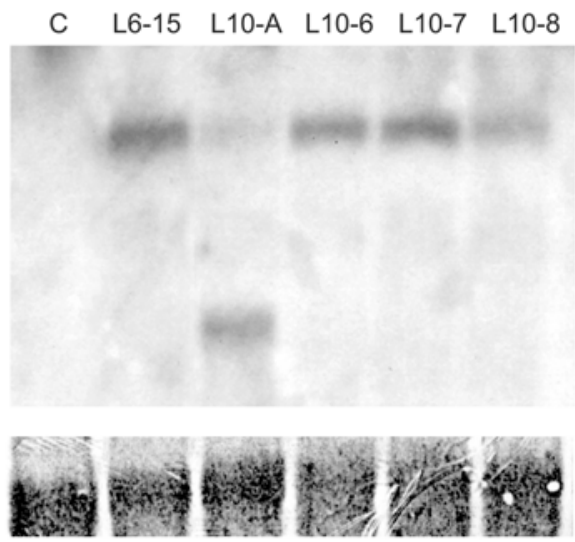

Fig. 2. Molecular analysis of the stunted L10-A plant. A, Gel blot analysis of a normal L10 transgenic plant (N) and the abnormal plant L10-A (A). Plant DNA was cut with $E c o R I$ and with $E c o$ RI and $B g l I I$ together. The full-length and truncated $L 10$ genes were detected using a probe that hybridizes to the $L 10$ promoter. The larger $E c o$ RI fragment is the $L 10$ gene that was cloned as an $E c o$ RI fragment into the transformation vector. Sizes of DNA markers $(\lambda \mathrm{H})$ are indicated. B, RNA gel blot analysis of $L 6$ and $L 10$ message in transgenic tobacco plants. Lane C (control) contains RNA from nontransgenic W38 tobacco; RNA in the remaining lanes is from L6-15, a stunted L6 transgenic, the stunted L10-A, and normal plants L10-6, L10-7, and L10-8. The lanes were loaded with equal amounts of RNA $(10 \mu \mathrm{g})$, and DNA from exon 1 and 2 of L10 was used as a ${ }^{32}$ P-labeled probe. The efficiency of transfer of RNA was monitored by staining the nylon filter with $0.2 \%$ methylene blue. 
mately 17 residues upstream from the P-loop of L10) and at least $120 \mathrm{C}$-terminal residues encoded by the retrotransposonlike gene (data not shown). The remainder of the open reading frame was not recovered in the clone. The presence of the $L 10$ fusion fragment in the genome L10-A was confirmed by PCR amplification of the appropriate size fragment, using a primer from $L 10$ and a primer from the retrotransposon-like sequence (data not shown).

To further investigate whether the truncated $L 10$ region is involved in the stunted phenotype, we made two expression vectors, L10short and 35SL10short, using the L10 and CaMV $35 \mathrm{~S}$ promoters, respectively, and containing just the $L 10$ sequences present in the truncated gene, with a stop codon introduced after the last $L 10$ codon in the cloned fragment (Fig. 3A). When either construct was expressed transiently by infiltration of an Agrobacterium strain into tobacco leaves, a strong necrotic reaction was induced (Fig. 3B and C). This reaction occurred when the binary vectors were in the Agrobacterium strain GV3101 pMP90 (Koncz and Schell 1986), which carries virulence genes necessary for T-DNA transfer, but not in the Ti plasmid-cured strain C58C1, which lacks these virulence genes. This indicated that the necrotic reaction depended on transfer of the T-DNA to plant cells. Furthermore, the reaction was specific to the deleted $\mathrm{L1O}$ construct. Controls using GV3101 pMP90 carrying the empty vector, genomic clones of $L 6$ and $L 10$, or an $L 10$ coding region with no introns and expressed using the $35 \mathrm{~S}$ promoter did not induce necrosis. These data are consistent with the postulate that the stunted phenotype of L10-A is due to the presence of the truncated $L 10$ gene. However, when these constructs were transformed into tobacco, none of the plants recovered showed any obvious phenotype similar to L10-A. The failure to recover transgenic plants that express a stunted phenotype similar to that of the original exceptional plant may be due to possible lethality of the transgene (Fig 3) or because the phenotype of L10-A is due to an interaction between the fulllength and truncated versions of $L 10$ in this plant. This was not investigated further.

\section{Stunted plants express a constitutive defense response.}

The stunted phenotype of the $L 6$ tobacco plants and L10-A is similar to that reported for mutants in resistance genes or resistance signaling genes that express constitutive defense responses, such as PR protein genes (Petersen et al. 2000, Shirano et al. 2002). Consequently, we examined the expression of three PR protein genes by RNA gel blot analysis and measured the activities of the phenylalanine ammonia lyase (PAL) and peroxidase enzymes in wild-type and L6 tobacco plants. RNA samples extracted from normal and stunted plants grown in tissue culture and in soil were analyzed for the abun-

A
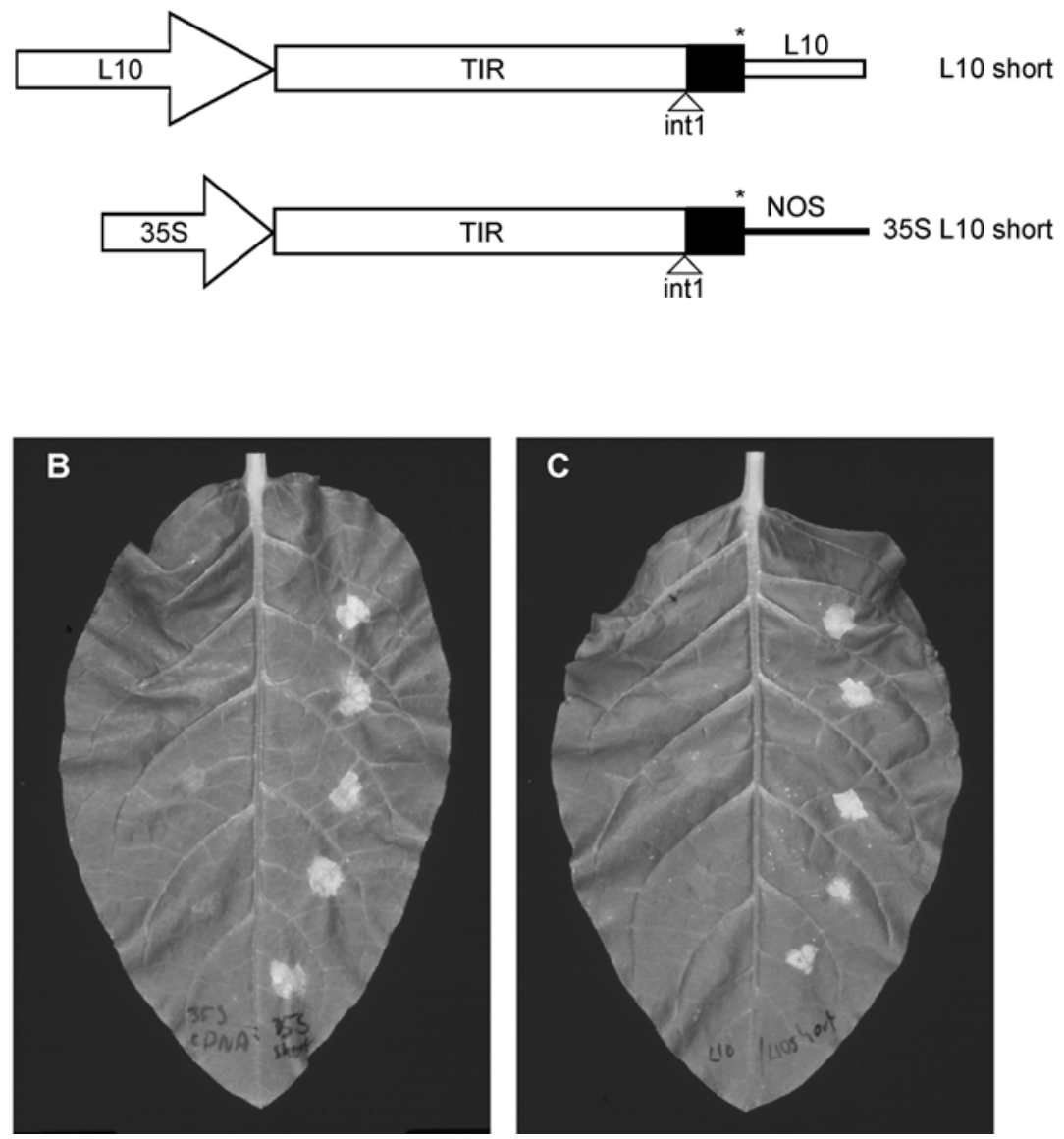

Fig. 3. Analysis of the synthetic truncated $L 10$ gene by transient assay in tobacco leaves. A, Constructs carrying the truncated $L 10$ gene containing exon 1 (TIR), intron 1 (int1), 39 codons of exon 2, and an introduced stop codon $\left(^{*}\right)$ were expressed using the L10 promoter and $3^{\prime}$ control region (L10 short) or 35S promoter and NOS 3' control region (35SL10 short). B, and C, Examples of transient expression analysis of the transgenes after syringe-infiltration of Agrobacterium cells into 1-cm diameter regions of tobacco leaves. In B, the left side of the leaf was infiltrated with a construct containing a full-length cDNA copy of the complete $L 10$ gene expressed by the $35 \mathrm{~S}$ promoter, and the right side was infiltrated with $35 \mathrm{SL} 10$ short. In C, the construct used on the left was the full-length $L 10$ genomic clone that was used for stable transformation, and the construct used on the right was L10 short. Necrosis was evident after 7 days and maximal at 14 days. Leaves were photographed 3 weeks postinfiltration. 
dance of three PR protein transcripts. Both PR1 (Fig 4) and PR2 (not shown) transcripts were abundant in L6 plants but not detected in the W38 controls or transgene-negative segregants. In contrast, PR3 was expressed in all plants with no obvious differences between $L 6$ and control plants (data not shown). Similar results were obtained from plants grown in soil. The exceptional L10 transgenic line, L10-A, also expressed PR1 transcripts but was not tested for expression of other defense genes (Fig. 4). The L10 transgenics that showed no stunting did not express PR1. PAL and peroxidase enzyme assays were determined for $L 6$ plants growing in soil. Increased peroxidase activity of six- and twofold control levels in lines J6-1 and J96-3 ( $P<0.0001$ and $P<0.002$, respectively) were detected. PAL activity, however, was not significantly different from equivalent untransformed tissues.

\section{$L 6$ plants have increased resistance to fungal pathogens.}

The effect of the $L 6$ gene on flax rust resistance in tobacco cannot be assessed, because tobacco is not a host for flax rust. Therefore the transgenic $L 6$ plants and control plants were grown in soil in a glasshouse and were assayed for their response to two tobacco pathogens, Phytophthora parasitica pv. nicotianae and Cercospora nicotianae. Inoculations were performed on $\mathrm{T}_{1}$ plants from two selected independent transgenic lines, JL96.3 and J6-1, in controlled-environment rooms, using conditions that favored optimal disease development. Homozygous and hemizygous transgenics were not distinguished in these experiments. The controls were W38 tobacco and nontransgenic segregants identified among selfed progeny of JL96.3 and J6-1 on the basis of their normal growth phenotype and failure of explants to form callus in tissue culture medium containing spectinomycin.

The resistance tests were complicated by the fact that the control and transgenic plants had different growth rates, so two experiments were done, the first comparing plants of the same age and the second comparing plants at the same developmental stage, achieved by delayed plantings of the nontrans-

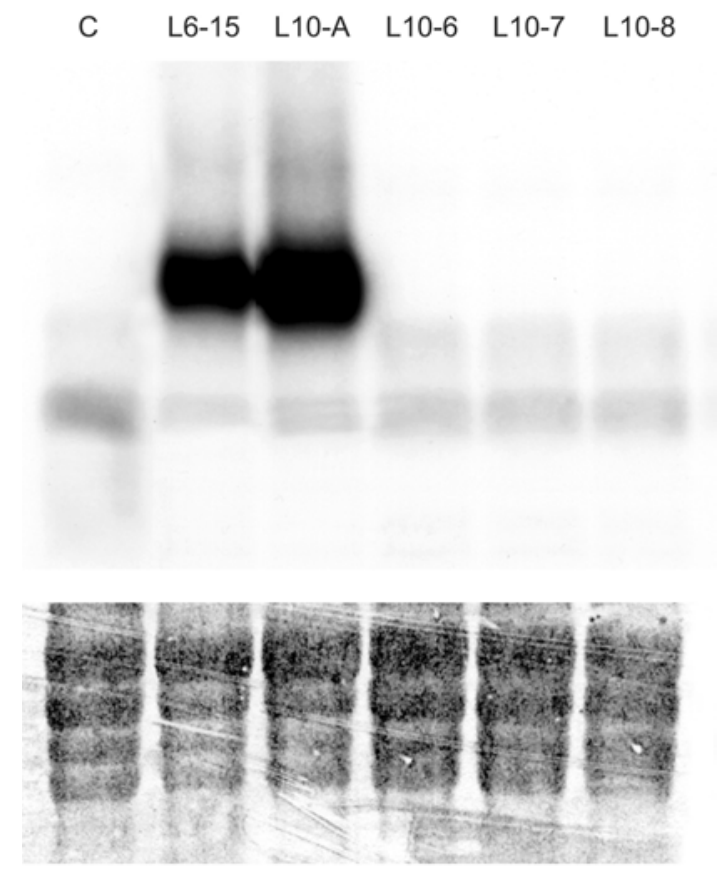

Fig. 4. PR1 gene expression in W38 and transgenic tobacco. RNA $(10 \mu \mathrm{g})$ from W38 (C), the stunted L6 plant L6-15, the stunted L10 plant L10-A, and three normal $L 10$ trangenics were separated by gel electrophoresis and were blotted onto nylon membrane (loading control, bottom) and probed with a ${ }^{32} \mathrm{P}$-labeled PR1 probe. genic plants. All experiments were done while plants were in a vegetative stage of growth. Ten-week-old plants ( 20 from each L6 lineage and the nontransgenic control) were decapitated, and the wounds were inoculated with $P$. parasitica pv. nicotianae. The lesion length on the inoculated stems was measured every 7 days over a period of 28 days after infection. Lesion length continued to increase each week in the controls, from 9 $\mathrm{cm}$ after 1 week to $20 \mathrm{~cm}$ at the end of the 4 -week trial. Lesion lengths were greatly reduced in the transgenic plants throughout the trial. In the first week, lesions had extended to $3.1 \mathrm{~cm}$ in the J6-1 transgenic progeny and spread less than $1 \mathrm{~cm}$ to 3.9 $\mathrm{cm}$ in the following 3 weeks (Fig. 5A). The average lesion lengths of both $L 6$ lineages were significantly different than the controls $(P<0.002)$ for the duration of the experiment. However, although the W38, J6-1, and JL96-3 plants were the same age, they exhibited vastly different average heights (31, 15 , and $9 \mathrm{~cm}$, respectively), due to the stunted phenotype associated with the $L 6$ transgene.

In a second experiment the decapitated stem inoculation was repeated using plants of the same height $(25 \mathrm{~cm})$, achieved by delayed plantings of wild-type seed. Thus the $L 6$ transgenic plants were two months older than the transgene-negative segregant controls. Plants (30 from each L6 lineage and the nontransgenic control) were decapitated and inoculated with $P$. parasitica pv. nicotianae. Lesion lengths were again reduced throughout the trial in the $L 6$ transgenics. Lesion length in the control line extended to $19.7 \mathrm{~cm}$ at the end of the 4-week trial. J6-1 and JL96-3 transgenic progeny reached 10.7 and $13.8 \mathrm{~cm}$, respectively (Fig. 5B). The average lesion lengths for both transgenic lineages were significantly different than the controls for all but one timepoint during the experiment. ( $\mathrm{P}$ values for the J6-1 line at $2[P<0.001], 3[P<0.003]$, and 4 $[P<0.007]$ weeks postinfection; for the JL96-3 line at $2[P<$ 0.006], $4[P<0.03]$, and $4[P<0.06]$ weeks postinfection). JL96-3 and J6-1 transgenic progeny did not differ significantly from one another for the duration of the experiment.

Resistance to $P$. parasitica pv. nicotianae was also tested using detached leaves. The third emergent leaf of four-week-old plants was selected for inoculations. A mycelial plug was placed over a wound site made in an interveinal region. Lesion area was measured daily for 4 days using the ASSESS system (Tucker Chakraborty 1997). Lesion development was significantly retarded in $L 6$-expressing plants compared with the nontransgenic control (J6-1: $P<0.01$, JL96-3: $P<0.006$ ) for the whole experiment (Fig. 5C).

Resistance to $C$. nicotianae, an important fungal pathogen of tobacco causing well-defined lesions, was also tested. The response of transgenic plants to this pathogen was evaluated by inoculating leaves from 8 -week-old $\mathrm{T}_{1} L 6$ transgenic progeny with fungal conidiophores and comparing percentage of leaf area infected (lesion area) to that of the nontransgenic control. There was significant reduction of disease development in both of the $L 6$ lineages where $P<0.0001$ for J6- 1 and $P<0.02$ for JL96-3 (Fig. 5D).

\section{DISCUSSION}

We have demonstrated that expression of $L 6$ from flax in transgenic tobacco gives rise to a stunted phenotype and increased but not complete resistance to two tobacco pathogens. It is likely that the observed level of resistance to the tobacco pathogens is due to constitutive expression of defense genes rather than recognition of the pathogens by the $L 6$ gene product, but we cannot rule out that increased resistance in the stunted transgenic plants was due to a combination of size differences on one hand and age differences on the other, which could not be eliminated in our experimental design. The 
stunting effect requires a wild-type P-loop in the NBS region of L6, which suggests that it is caused by the intrinsic activity of the L6 resistance protein. The reason $L 6$ but not the closely related $L 2$ and $L 10$ alleles induces plant stunting and defense gene activation is unknown. Although the differences do not appear to derive from different expression levels of the alleles (Fig. 2B), they could be due to posttranscriptional effects, including mRNA processing due to DNA sequence differences between the alleles or other factors associated with sequence differences of the three gene products that affect specificity or protein stability.

One transgenic, L10-A, which expressed an extreme phenotype, was an exception to the general observation that tobacco expressing L10 had a wild-type appearance. The phenotype of this $\mathrm{T}_{0}$ plant and its progeny was associated with the presence of a truncated $L 10$ gene that encodes a predicted fusion protein consisting of the TIR region and 39 amino acids from the beginning of the NBS domain of L10 and a retrotransposon protein. Although a synthetic gene consisting of just the L10 regions induced necrosis when expressed transiently in tobacco leaves, the stunted phenotype was not observed in plants selected from stable transformation experiments. Based on the necrotic phenotypes in the transient assay and the extreme phenotype of L10-A, there could have been strong selection during tissue culture against regenerating transgenic plants expressing this gene. Additionally, the L10-A phenotype may derive from the simultaneous presence of full-length and truncated L10 in the L10-A plant. It has been postulated that the TIR region of resistance proteins has a signaling role in disease resistance (Whitham et al. 1994), but no experimental evidence is available supporting this idea. It is possible that the phenotype of the L10-A plant and the transient expression phenotype are the result of constitutive TIR region signaling by the truncated protein that is normally repressed in the full-length L10 protein. Additional investigation of this TIR region-only effect, particularly in Arabidopsis in which various potential signaling pathway mutants for TIR-NBS-LRR resistance genes are known, could shed further light on the function of the TIR region in disease resistance proteins.

The molecular basis for the induction of defense responses and the associated stunted phenotype in tobacco by $L 6$ is unknown. However, experiments involving overexpression of wild-type NBS-LRR genes (Oldroyd and Staskawicz 1998; Stokes et al. 2002) or expression of mutant NBS-LRR genes, like the Potato virus $X$ resistance gene $R x$ in Nicotiana bethamiana (Bendahamane et al. 2002, Moffet et al. 2002) or SSI4 in Arabidopsis (Shirano et al. 2002), provide some ideas. In all these examples, overexpression or particular mutations give rise to pathogen-independent defense gene expression and, often, an associated stunted plant phenotype. The causal link between
A

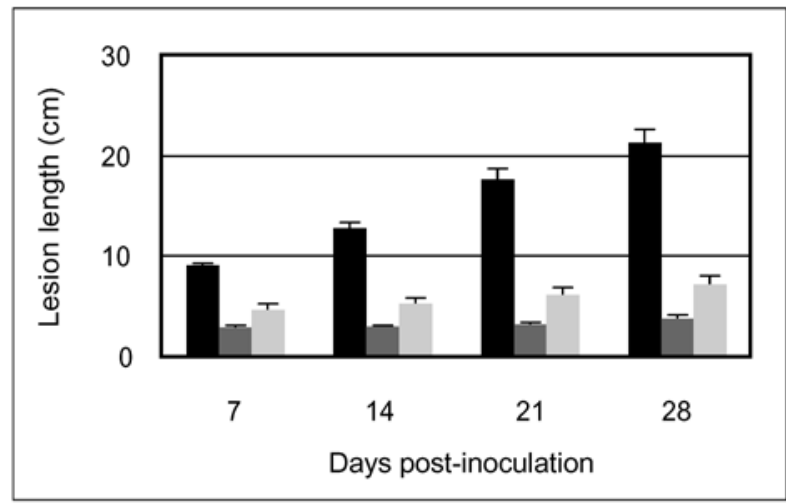

C

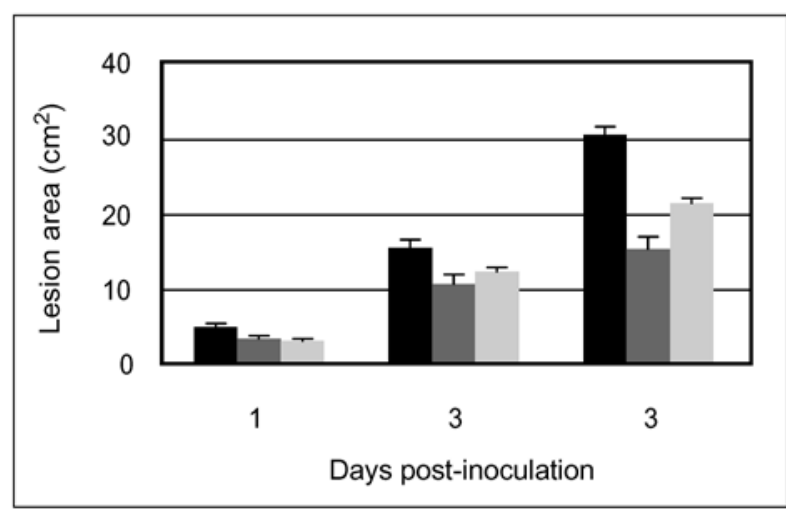

B

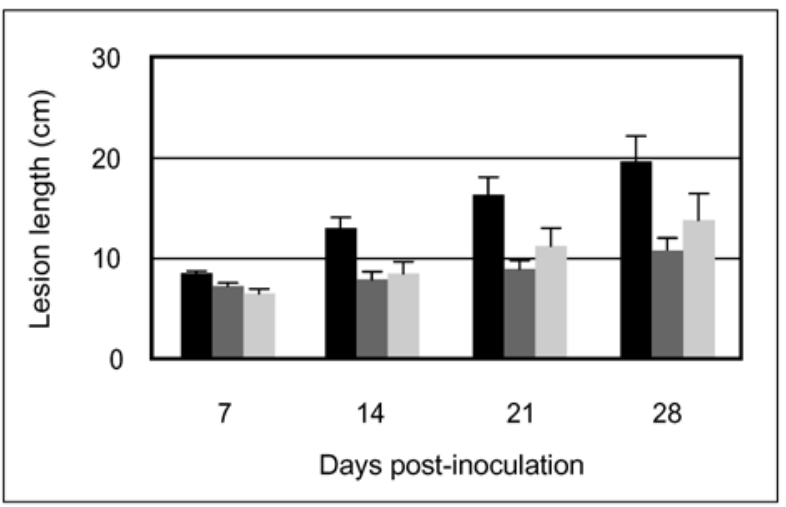

D

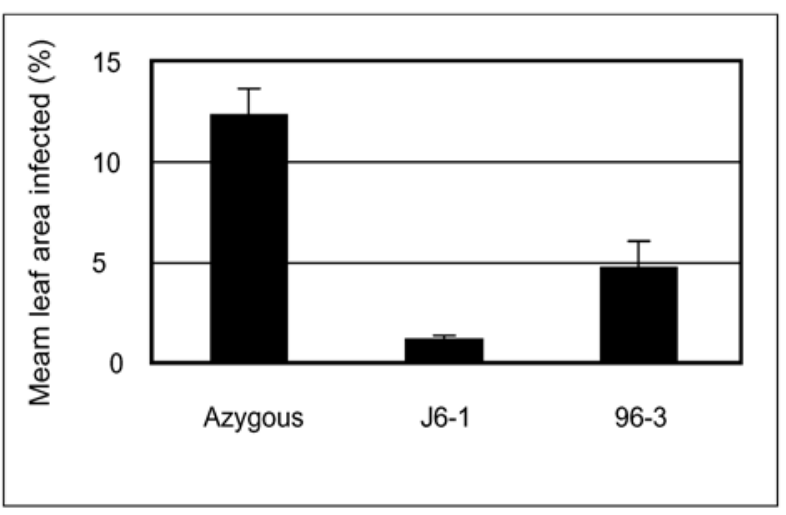

Fig. 5. L6 tobacco transgenics have increased disease resistance. A, Disease progression on decapitated stems of 10-week-old control and $L 6$ lines inoculated with Phytophthora parasitica pv. nicotianae. Controls were $\mathrm{T}_{1}$ segregants that did not carry L6. Bars are means with standard error from 20 plants per line. B, Disease development on decapitated stems of $\mathrm{T}_{1}$ control and $L 6$ lines that were the same height at time of inoculation with $P$. parasitica pv. nicotianae. Bars are means with standard error from 20 plants per line. C, Detached leaves of control and L6 lines inoculated with P. parasitica pv. nicotianae. Bars represent means with standard error on 20 leaves per line. D, Disease severity lesion area on $\mathrm{T}_{1}$ control and transgenic lines expressing $L 6$ 12 days postinoculation with Cercospora nicotianae. Bars represent means with standard error on 20 plants per line. 
constitutive defense gene expression and the stunted phenotype is unknown. Nevertheless, the data in all these papers suggest that NBS-LRR proteins are normally in an inactive state and that they are activated by pathogen stimuli that then lead to signaling, defense gene expression, and disease resistance. Mutation-induced activation could be due to amino acid substitutions that cause a conformational change or the modification of a binding site preventing interaction with a hostderived repressor protein, or both. Overexpression of the wildtype protein, which may have some intrinsic activity, could result in sufficient protein to induce detectable defense responses. Overexpression of the wild-type protein could also lead to titration of a second protein that normally represses resistance protein activation (Oldroyd and Staskawicz 1999; Stokes et al. 2002). The Arabidopsis protein RIN4 has been identified as a repressor of the activity of the resistance protein RPS2 (Axel and Staskawicz 2003; Mackey et al. 2003). When the effector protein avrRPT2 is delivered to the plant cell by Pseudomonas syringae, RIN4 is degraded and RPS2 becomes active. RIN4 also is the "virulence target" of a second bacterial effector protein, avrRPM1, which induces the phosphorylation of RIN4. It is postulated that the resistance protein RPM1 is activated by phosphorylated RIN4. In light of these recent findings, we consider the following possibilities for the activation of tobacco defenses by the $L 6$ transgene: i) compared with flax, the expression levels of L6 protein may be higher in tobacco, leading to a dose-dependent defense activation, ii) flax but not tobacco produces a protein, equivalent in action to RIN4, that represses L6 protein activity in the absence of pathogens, iii) tobacco but not flax expresses a protein equivalent in action to phosphorylated RIN4 that activates L6 but not related tobacco resistance proteins in the absence of pathogens, or iv) tobacco itself may recognize the nonmutant form of L6 as "foreign" and activate defense responses. This situation could arise if L6 titrated a tobacco repressor protein that normally maintained tobacco resistance proteins in an inactive state in the absence of pathogens. All of these possibilities may influence the outcomes of cross-species transfers of plant disease resistance genes for crop protection.

\section{MATERIALS AND METHODS}

\section{Transformation of tobacco.}

Tobacco cv. W38 was transformed as described previously (Ellis et al. 1987), except that the selectable marker gene used encoded spectinomycin resistance (Svab et al. 1990). Shoots were recovered from inoculated leaf disks after selection on spectinomycin ( $200 \mathrm{mg} / \mathrm{liter})$, were transferred to a hormonefree medium for root induction, and then, were transferred singly to tissue culture pots. These plants were confirmed as transgenic by the ability of leaf pieces from each plant to form callus and regenerate shoots in the presence of spectinomycin (200 mg/liter). The Agrobacterium strains containing binary vectors carrying the genomic copies of the $L 2, L 6$, and $L 10$ genes used for transformation of tobacco have been described previously (Ellis et al. 1999). The Agrobacterium strain containing the binary vector carrying the $35 \mathrm{~S} L 6 \mathrm{cDNA}$ gene (no introns) was also described previously (Ayliffe et al. 1999). Previous transformation experiments with these strains in flax had confirmed the resistance function of the clones (Ayliffe et al. 1999; Ellis et al. 1999).

\section{Gene silencing.}

An inverted repeat/intron silencing vector for the $L 10$ gene was constructed, using the general strategy described by Smith and associates (2000). The construct included the exon 1 region of $L 10$, which was amplified from a plasmid clone of the $L 10$ gene (Ellis et al. 1999) by PCR, using the primers L10-ex1A (5'GGGGTCTAGACTCGAGTTGCTACTGCTGTTGCCTTG-3') and L10-Ex1B (5'-CGGGGAAGCTTGAATTCGTCATCCTTT CCGATGTGCC- $3^{\prime}$ ) The cloned PCR product was digested with restriction enzymes in two separate reactions, the first with XhoI and EcoRI and the second with XbaI and HindIII. These sites occur in the primer sequences. The PCR product was then inserted into vector pN6 (Smith et al. 2002) in sequential cloning steps. In the first cloning step, the XhoI/EcoRI fragment was inserted into pN6, then the XbaI/HindIII fragment was inserted into the product of this ligation. This created an inverted repeat of the $L 10$ exon 1 region, separated by an intron and expressed using the $35 \mathrm{~S}$ promoter. After confirmation by DNA sequencing, the inverted repeat gene was cloned as a NotI fragment into the binary vector pART27 (Smith et al. 2002). This vector was transferred to the Agrobacterium strain GV3101pMP90 (Koncz and Schell 1996). The resulting Agrobacterium strain was used to super-transform spectinomycin-resistant stunted (putative hemizygous) $T_{1}$ progeny derived from the selfing of the $L 10$ tobacco line that expressed the stunted phenotype. Super-transformed plants were recovered by selection on kanamycin sulphate (100 mg/liter).

\section{Cloning the truncated $L 10$ gene.}

The truncated $L 10$ gene and flanking plant DNA was cloned by long-range PCR, using the gene walker system described by Siebert and associates (1995). DNA from the tobacco plant carrying $L 10$ and expressing a stunted phenotype was digested with $B g l I I$ (DNA gel blot analysis had indicated that the truncated copy of $L 10$ was associated with an approximately 3-kbp $B g l I I$ fragment). The $B g l I I-d i g e s t e d$ DNA was ligated with the adapters described by Siebert and associates (1995), except that the sequence of short oligonucletides was changed so that the adapter ligated to the $B g l I I$ ends of the restricted plant DNA. The ligated DNA was amplified, using primer pairs L10-1 and ap1 and was reamplified using primer pairs L10-2 and ap2 after 100-fold dilution of the first PCR product. Primers ap1 and ap2 were described by Siebert and associates (1995), L10-1 was 5'-GCTGATAGTAAGTGGTGTCTTATGG AGCTG-3', and L10-2 was 5'-AGAGGAGGACCCTCGACG CATCATACTTCC-3'. These sequences occur $5^{\prime}$ to a BamHI site in exon 1 of L10. The PCR product was digested with NotI and BamH1, was cloned into pUC119, and was sequenced.

\section{Leaf infiltration experiments.}

Agrobacterium strains carrying various binary vector plasmids were grown overnight in Luria-Bertani medium plus $5 \mathrm{mg}$ of tetracycline per liter to maintain the binary plasmid. The cells were centrifuged, washed, and resuspended in MurashigeSkoog medium at an optical density at $600 \mathrm{~nm}$ of 0.5 or 1.0 in different experiments, and then, using a $20 \mathrm{ml}$ plastic syringe (no needle), were infiltrated into the lower surface of five consecutive leaves, starting with the largest leaf on approximately 20 - to 30 -cm-high tobacco plants (cv. Samsun) in $15-\mathrm{cm}$ pots, to produce infiltrated zones of approximately 1 $\mathrm{cm}$ in diameter. Necrosis started to appear after 1 week and developed to a maximum by 14 days, and leaves were scored and photographed 2 or 3 weeks after infiltration. From the bottom of the plant, there was a clear gradient in sensitivity; leaves 1 and 2 often gave no and little reaction, respectively, leaves 3 and 4 gave a maximum reaction, and leaf 5, which was small at the time of infiltration and difficult to infiltrate, often gave a weak reaction.

\section{DNA, RNA, and enzyme analysis.}

Tobacco DNA extraction and gel blot analysis was done as previously described (Anderson et al. 1997). Expression of $L$ 
genes in transgenic tobacco was detected by RNA gel blot analysis using a probe derived from the exon 1 and 2 regions of $L 10$ or by RT-PCR as described by Ayliffe and associates (1999), or both. RNA transfer to nylon membranes was monitored by staining the gel with $0.2 \%$ methylene blue dissolved in $0.2 \mathrm{M} \mathrm{Na}$ acetate $(\mathrm{pH} \mathrm{5.2)}$ for $30 \mathrm{~s}$, followed by several rinses in water to remove excess color. Expression of tobacco PR proteins was detected by standard RNA gel blot procedures, using probes from the coding regions of tobacco PR1, PR2, and PR3. PAL and peroxidase assays were carried out as described (Way et al. 2000, 2002).

\section{Pathogen infection studies and statistical analysis.}

Isolate UQ2772 of Phytophthora parasitica pv. nicotianae was obtained from the culture collection of the Cooperative Research Center for Tropical Plant Pathology, University of Queensland, Brisbane, Australia. Cultures were maintained on V8 medium (250 ml V8 broth, $3.5 \mathrm{~g} \mathrm{CaCo}_{3}, 2 \%$ Bacto Agar [Difco, Detroit]) under near-UV light at $25^{\circ} \mathrm{C}$. The Cercospora nicotianae isolate was obtained from P. Trevorrow, Department of Primary Industries, Mareeba, Queensland, Australia. C. nicotianae was grown on V8 medium (as for $P$. parasitica pv. nicotianae) with the addition of ground tobacco leaves (100 g per 1iter of media) and supplemented with $15 \mu \mathrm{g}$ of tetracycline per $\mathrm{ml}$ and $100 \mu \mathrm{g}$ of streptomycin per $\mathrm{ml}$. Cultures were maintained under near-UV light at $25^{\circ} \mathrm{C}$.

Disease trials were conducted in controlled-environment rooms (16-h photoperiod, a light intensity of $500 \mu \mathrm{mol} \mathrm{m} \mathrm{m}^{-2}$ ). Inoculations with the oomycete $P$. parasitica $\mathrm{pv}$. nicotianae were performed using two different methods based on those described by Robin and Guest (1994). A decapitated-stem technique and a detached leaf assay were used. In the decapitated-stem experiments, plants were incubated at a temperature regime of 27 and $25^{\circ} \mathrm{C}$ for day and night, respectively, with $95 \%$ relative humidity. The fungus was cultured on V8 agar at $25^{\circ} \mathrm{C}$ for three days under near-UV light. A 4-mm agar mycelial plug, taken from the outer perimeter of the growing hyphal front, was inverted and placed on the cut site of decapitated stems of 8-week-old plants. An Eppendorf tube to hold the agar plug in place and maintain humidity then covered the infection site. The stem lesion extension was measured every 7 days for 28 days. Mean lesion length was an average from 20 plants for each of the transgenic and azygous control lines. The second method of inoculating tobacco with $P$. parasitica pv. nicotianae involved detaching and infecting the fourth leaf from 4-week-old plants. The leaf was placed abaxial side up on filter paper wetted with $1 \mathrm{ml}$ of benzimidazole per 1 iter in $17-\mathrm{cm}$ petri dishes. A small cut with a scalpel was made in the interveinal region of the leaf, and a 4$\mathrm{mm}$ agar mycelial plug was inverted and placed on the cut site. Petri dishes were incubated in a tissue-culture facility with 16$\mathrm{h}$ day length and a temperature regime of $28^{\circ} \mathrm{C}$ constant. Leaves (20 each) from both transgenic lines and the nontransgenic control line were used for each experiment. Lesion area was measured daily for 4 days, using ASSESS, a digital capture and software analysis system (Tucker and Chakraborty 1997).

Inoculations with the fungus $C$. nicotianae (Frog eye disease) were undertaken on 6-week-old plants. The inoculum was prepared from 5-day cultures at a concentration of $6 \times 10^{4}$ conidiophores per $\mathrm{ml}$. The leaves were sprayed till run-off, and the plants were enclosed in polyethylene bags for $48 \mathrm{~h}$. Infections were undertaken at $28^{\circ} \mathrm{C}$-day and $25^{\circ} \mathrm{C}$-night temperatures with $95 \%$ relative humidity. A total of 20 plants were inoculated for each transgenic and control line. Disease severity was rated 12 days after inoculation, by comparison to a computer-generated disease assessment key.
For all experiments, plants were arranged randomly before inoculation. Each day's results were analyzed separately. Data were tested for the assumption of normality using the AndersonDarling test. If necessary, detected outliers were eliminated or the variable was transformed prior to analysis. Levene's test was then used to test for homogeneity of variances.

Data for $P$. parasitica pv. nicotianae inoculation experiments were analyzed using one way analysis of variance to compare the lines, including the azygous control each day. A significant result for the model was followed by Dunnett's one-tail post hoc test to see whether there was a significant improvement in any "treated" line compared with the control. The data were reanalyzed after excluding the control, and a significant result for the model was followed by Tukey's pairwise post hoc test to see whether there were any significant differences between the treated lines $(P<0.05)$. Data for the "percentage leaf area infected" following $C$. nicotianae were not normally distributed. The null-hypothesis of no association of line with "leaf area infected (\%)" was tested using the "maximum likelihood" chi-square statistic and Monte-Carlo randomization was used to determine significance.

\section{ACKNOWLEDGMENTS}

L. Thorpe and V. Ryle are thanked for excellent technical assistance.

\section{LITERATURE CITED}

Anderson, P. A., Lawrence, G. J., Morrish, B. C., Ayliffe, M. A., Finnegan, E. J., and Ellis, J. G. 1997. Inactivation of the flax rust resistance gene $M$ associated with loss of a repeated unit within the leucine-rich repeat coding region. Plant Cell 9:641-651.

Axtell, M. J., and Staskawicz, B. J. 2003. Initiation of RPS2-specified disease resistance in Arabidopsis is coupled to the AvrRpt-2directed elimination of RIN4. Cell 112:369-377.

Ayliffe, M. A., and Frost, D. V., Finnegan, E. J., Lawrence, G. J., Anderson, P. A., and Ellis J. G. 1999. Analysis of alternative transcripts of the flax L6 rust resistance gene. Plant J. 17:287-292.

Bendahamane, A., Farnham, G., Moffett, P., and Baulcombe D. C. 2002. Constitutive gain-of-function mutants in a nucleotide binding siteleucine rich repeat protein encoded at the $R x$ locus of potato. Plant J. 32:195-204.

Dinesh-Kumar, S. P., Tham, W. H., and Baker, B. J. 2000. Structurefunction analysis of the tobacco mosaic virus resistance gene $N$. Natl Acad. Sci. U.S.A. 97:14789-14794.

Ellis, J. G., Llewellyn, D. J., Dennis, E. S., and Peacock, W. J. 1987. Maize Adh-1 promoter sequences control anaerobic regulation: Addition of upstream promoter elements from constitutive genes is necessary for expression in tobacco. EMBO (Eur. Mol. Biol. Organ.) J. 6:3203-3208.

Ellis, J. G., Lawrence, G. J., Luck, J. E., and Dodds, P. N. 1999. Identification of regions in alleles of the flax rust resistance gene $L$ that determine differences in gene-for-gene specificity. Plant Cell 11:495-506.

Hennin, C., Hőfte, M., and Diederichsen, E. 2001. Functional expression of Cf9 and Avr9 genes in Brassica napus induces enhanced resistance to Leptosphaeria maculans. Mol. Plant-Microbe Interact. 14:1075-1085.

Koncz, C., and Schell, J. 1986. The promoter of $\mathrm{T}_{\mathrm{L}}$-DNA gene 5 controls the tissue specific expression of the chimaeric genes carried by a novel type of Agrobacterium binary vector. Mol. Gen. Genet. 204:383-396.

Lawrence, G. J., Finnegan,, E. J., Ayliffe, M. A., and Ellis, J. G. 1995. The L6 gene for flax rust resistance is related to the Arabidopsis bacterial resistance gene RPS2 and the tobacco viral resistance gene $N$. Plant Cell 7:1105-1206.

Mackey, D., Belkhadir, Y., Alonso, J. M., Ecker, J. R., and Dangl, J. L. 2003. Arabidopsis RIN4 is a target of the type III virulence effector AvrRpt2 and modulates RPS2-mediated resistance. Cell 112:379-389.

Moffett, P., Farnham, G., Peart, J., and Baulcombe, D. C. 2002. Interaction between domains of a plant NBS-LRR protein in disease resistancerelated cell death. EMBO (Eur. Mol. Biol. Organ.) J. 21:4511-4519.

Oldroyd, G. E. D., and Staskawicz, B. J. 1998. Genetically engineered broad-spectrum disease resistance in tomato. Proc. Natl. Acad. Sci. U.S.A. 95:10300-10305.

Petersen, M., Brodersen, P., Naested, H., Andreasson, E., Lindhart, U., Johansen, B., Nielsen, H. B., Lacy, M., Austin, M. J., Parker, J. E., 
Sharma, S. B., Klessig, D. F., Martienssen, R., Mattsson, O., Jensen, A B., and Mundy, J. 2000. Arabidopsis map kinase 4 negatively regulates systemic acquired resistance. Cell 103:1111-1120.

Robin, C., and Guest, D. 1994. Characterisation of pathogenicity of Phytophthora parasitica isolates by stem and detached-leaf inoculations in four tobacco cultivars. N. Z. J. Crop Hortic. Sci. 22:159-166.

Siebert, P. D., Chenchik, A., Kellog, D. E., Lukyanov, K. A., and Lukyanov, S. A. 1995. An improved PCR method for walking in uncloned genomic DNA. Nucleic Acids Res. 23:1087-1088.

Shirano, Y., Kachroo, P., Shah. J., and Klessig, D. F. 2002. A gain-offunction mutation in an Arabidopsis toll interleukin1 receptor-nucleotide binding site-leucine-rich repeat type $R$ gene triggers defense responses and results in enhanced disease resistance. Plant Cell 14:3149-3162.

Smith, N. A., Singh, S. P., Wang, M. B., Stoutjesdijk, P. A., Green A. G., and Waterhouse, P. M. 2000. Total silencing by intron-spliced hairpin RNAs. Nature 407:319-320

Stokes, T. L., Kunkel, B. N., and Richards E. J. 2002. Epigenetic variation in Arabidopsis disease resistance. Genes Dev. 16:171-182.

Svab, Z., Harper, E. C., Jones, J. D., and Maliga, P. 1990. Aminoglycoside-3"-adenyltransferase confers resistance to spectinomycin and streptomycin in Nicotiana tabacum. Plant Mol. Biol. 14:197-205.

Tai, T. H., Dahlbeck, D., Clark, E. T., Gajiwala, P., Pasion, R., Whalen, M. C., Stall, R. E., and Staskawicz, B. J. 1999. Expression of the Bs2 pepper gene confers resistance to bacterial spot disease in tomato. Proc. Natl. Acad. Sci. U.S.A. 96:14153-14158.

Tang, X., Xie, M., Kim, Y. J., Zhou, J., Klessig, D. F., and Martin, G. B. 1999. Overexpression of Pto activates defense responses and confers broad resistance. Plan Cell 11:15-29.

Tao, Y., Yuan, F., Leister, R. T., Ausubel, F. M., and Katagiri, F. 2000.
Mutational analysis of the Arabidopsis nucleotide binding site-leucinerich repeat resistance gene RPS2. Plant Cell 12:2541-2554.

Tornero, P., Chao, R. A., Luthin, W. N., Goff, S. A., and Dangl, J. L. 2002.

Large-scale structure-function analysis of the Arabidopsis RPM1 disease resistance protein. Plant Cell 14:435-450.

Tucker, C. C., and Chakraborty, S. 1997. Quantitative assessment of lesion characteristics and disease severity using digital image processing. J. Phytopathol. 145:273-278.

Way, H. M., Kazan, K., Goulter, K. G., Birch, R. G., and Manners, J. M. 2000. Expression of the Shpx2 peroxidase gene of Stylosanthes humilis in transgenic tobacco leads to enhanced resistance to Phytophthora parasitica pv. nicotianae and Cercospora nicotianae. Mol. Plant Pathol. 1: 223-232.

Way, H. M., Kazan, K., Mitter, N., Goulter, K. G., Birch, R. G., and Manners, J. M. 2002. Expression of the ShPAL phenylalanine ammonia lyase gene of Stylosanthes humilis in transgenic tobacco leads to enhanced disease resistance but impaired plant growth. Physiol. Mol. Plant Pathol. 60:275-282.

Whitham, S., Dinesh-Kumar, S. P., Choi, D., Hehl, R., Corr, C., and Baker, B. 1994. The product of the tobacco mosaic virus resistance gene $N$ : Similarity to toll and the interleukin-1 receptor. Cell 78:11011115 .

Whitham, S., McCormick, S., and Baker, B. 1996. The $N$ gene of tobacco confers resistance to Tobacco mosaic virus in transgenic tomato. Proc. Natl. Acad. Sci. U.S.A. 93:8776-8781.

Xiao, S., Charoenwattana, P., Holcombe, L., and Turner, J. G. 2003. The Arabidopsis genes $R P W 8.1$ and $R P W 8.2$ confer induced resistance to powdery mildew diseases in tobacco. Mol. Plant-Microbe Interact. 16:289-294 